\title{
Prevalence, putative mechanisms, and current management of sleep problems during chemotherapy for cancer
}

This article was published in the following Dove Press journal:

Nature and Science of Sleep

13 December 2012

Number of times this article has been viewed

\author{
Oxana Palesh' \\ Luke Peppone ${ }^{2}$ \\ Pasquale $\mathrm{F}$ Innominato ${ }^{3-5}$ \\ Michelle Janelsins ${ }^{2}$ \\ Monica Jeong' \\ Lisa Sprod ${ }^{7}$ \\ Josee Savard ${ }^{6}$ \\ Max Rotatori' \\ Shelli Kesler' \\ Melinda Telli' \\ Karen Mustian ${ }^{2}$ \\ 'Stanford University School of \\ Medicine, Stanford, CA, USA; \\ ${ }^{2}$ University of Rochester School of \\ Medicine and Dentistry, Rochester, \\ NY, USA; ${ }^{3}$ INSERM, UMRS 776, \\ Biological Rhythms and Cancers, \\ Villejuif, France; ${ }^{4}$ Faculty of \\ Medicine, Universite Paris Sud, le \\ Kremlin-Bicêtre, France; ${ }^{5} \mathrm{APHP}$, \\ Chronotherapy Unit, Department \\ of Oncology, Paul Brousse Hospital, \\ Villejuif, France; ' ${ }^{6}$ aval University, \\ Quebec, Canada; ${ }^{7}$ University of North \\ Carolina, Wilmington, NC, USA
}

Correspondence: Oxana Palesh 40I Quarry Road, Office 2318 Stanford, CA 94305-5718, USA

Tel + I 65072570 II

Fax + I 6504986678

Email opalesh@stanford.edu

\begin{abstract}
Sleep problems are highly prevalent in cancer patients undergoing chemotherapy. This article reviews existing evidence on etiology, associated symptoms, and management of sleep problems associated with chemotherapy treatment during cancer. It also discusses limitations and methodological issues of current research. The existing literature suggests that subjectively and objectively measured sleep problems are the highest during the chemotherapy phase of cancer treatments. A possibly involved mechanism reviewed here includes the rise in the circulating proinflammatory cytokines and the associated disruption in circadian rhythm in the development and maintenance of sleep dysregulation in cancer patients during chemotherapy. Various approaches to the management of sleep problems during chemotherapy are discussed with behavioral intervention showing promise. Exercise, including yoga, also appear to be effective and safe at least for subclinical levels of sleep problems in cancer patients. Numerous challenges are associated with conducting research on sleep in cancer patients during chemotherapy treatments and they are discussed in this review. Dedicated intervention trials, methodologically sound and sufficiently powered, are needed to test current and novel treatments of sleep problems in cancer patients receiving chemotherapy. Optimal management of sleep problems in patients with cancer receiving treatment may improve not only the well-being of patients, but also their prognosis given the emerging experimental and clinical evidence suggesting that sleep disruption might adversely impact treatment and recovery from cancer.
\end{abstract}

Keywords: cancer, sleep, chemotherapy, intervention, circadian rhythm, cognitive behavioral therapy

\section{Sleep problems in cancer}

Cytotoxic chemotherapy, along with other cancer treatments (surgery, radiotherapy, and hormonal and targeted therapies), has led to increases in survival rates for many types of cancer over recent decades. ${ }^{1}$ While chemotherapy is a highly effective cancer treatment, its administration is often associated with side effects that significantly reduce patients' quality of life. Some of the most common chemotherapy-related side effects include fatigue, depression, pain, nausea/vomiting, and sleep problems. ${ }^{2,3}$

Sleep problems - difficulty falling or staying asleep, poor sleep quality, and/or short sleep duration - are among the most frequently reported side effects resulting from cancer treatment. These sleep problems are considered to be symptoms of insomnia, and when several sleep problems co-occur and are severe, the cluster is defined as insomnia syndrome. If a patient does not meet the criteria for insomnia syndrome but has disrupted sleep, he or she is considered to have insomnia symptoms. Patients meet the criteria for insomnia syndrome if they have difficulty falling asleep or staying asleep, or 
if they wake up earlier than intended at least three times a week for longer than 30 minutes. This disturbance merits clinical attention if it lasts longer than 1 month. However, in cancer patients undergoing treatment sleep disturbance of shorter duration might also merit clinical attention. Insomnia symptoms and insomnia syndrome are the most prevalent sleep problems among cancer patients. ${ }^{4,5}$ For the purpose of consistency throughout this review, we will use the broader term "sleep problems" to represent both conditions.

\section{Prevalence of sleep problems}

The prevalence of sleep problems is much higher in cancer patients than in the general population; sleep problems are reported in $30 \%-87 \%$ of cancer patients. ${ }^{4-11}$

Among the sleep problems reported during chemotherapy, some of the most common complaints are frequent night awakenings (49\%-56\%), inability to fall asleep within 30 minutes (50\%-73\%), and early awakenings (49\%-65\%). ${ }^{14,15}$ In addition, after cancer diagnosis and before the initiation of chemotherapy, ${ }^{16,17}$ patients experience poor sleep quality and a number of sleep problems; thus, up to two thirds of patients on chemotherapy report impaired sleep.,11

\section{Sleep problems throughout the cancer trajectory}

While the prevalence of sleep problems is highest during chemotherapy, research shows that sleep problems continue through cancer survivorship and often persist for years after the completion of cancer therapy. ${ }^{18}$ One study reported that $57 \%$ of long-term ( $>5$ years) lung cancer survivors had poor sleep compared to $30 \%$ of non-cancer controls. ${ }^{19}$ Other studies support those results, showing higher rates of sleep problems and daytime sleepiness among cancer survivors compared to those without cancer. ${ }^{8,20,21}$ While the majority of studies of sleep problems in cancer patients are cross-sectional, a small number of prospective studies have examined sleep problems longitudinally. These studies show that throughout the cancer treatment process sleep problem rates are elevated and remain relatively stable., ${ }^{7,22}$ Other studies have found that the prevalence of sleep problems declines only slightly over time, following completion of anticancer treatment..$^{5,23-25}$ These prospective studies demonstrate that sleep problem rates remain elevated for years in cancer patients (those who are still undergoing cancer treatments) and continue to be a chronic condition that survivors (those patients who are finished with their cancer treatments) experience. Furthermore, sleep problems in cancer patients are frequently accompanied by other symptoms, most commonly fatigue, depression, and anxiety. ${ }^{7,15,26}$ Impairment of sleep is highly correlated with increased levels of fatigue and depression. ${ }^{27-29}$ Isolating the impact of chemotherapy on sleep remains difficult because the administration of chemotherapy varies as a function of disease type, stage, and clinical progression, and chemotherapy administered cyclically causes side effects (eg, nausea) that could precipitate the development of sleep problems. Regardless, studies consistently show that sleep impairment is a highly prevalent and significant issue among cancer patients that warrants further investigation and the development of effective treatments.

\section{Sleep assessment}

One of the methodological issues that should be considered when reviewing studies on sleep problems in cancer patients is the variety of instruments and questionnaires that have been used to assess sleep. The Pittsburgh Sleep Quality Index (PSQI), the Insomnia Severity Index (ISI), the Women's Health Initiative Insomnia Rating Scale, and the Epworth Sleepiness Scale are among the most prevalent validated sleep measures used in cancer patients. However, many researchers also have developed their own sleep questionnaires, and others have used a single item or selected ones from larger questionnaires assessing other constructs (eg, depression, quality of life). The two measures that have been successfully used to identify clinically significant sleep problems in cancer patients are the PSQI and the ISI. These measures have established clinical cut-offs that can identify clinically significant sleep problems.

In cancer patients a score of 8 or higher on the PSQI represents a clinical cut-off for identifying sleep problems that need treatment. ${ }^{29}$ A study ${ }^{30}$ on 1670 cancer patients showed that a score of 8 or higher on the ISI represents a clinical cut-off for identifying sleep problems that are severe enough to warrant treatment.

Objective measures of sleep have also been used in cancer patients. Studies utilizing actigraphy measurement (a wrist-worn device that measures locomotor activity counts) showed that chemotherapy disrupts sleep-wake cycles as early as the first cycle ${ }^{11}$ and that these disruptions tend to worsen as chemotherapy continues. ${ }^{31}$ Studies that have used the gold standard of sleep measurement, polysomnography monitoring, to assess sleep problems in cancer patients undergoing treatment have found conflicting evidence. The first, by Parker et al, involving 114 patients with various metastatic cancers, showed that patients had dysregulated sleep-wake cycles characterized by low sleep efficiency (77.2\%), light sleep (as measured by high prevalence of stages 1 and 2), and low prevalence of restorative slow-wave sleep. ${ }^{32}$ The second study, a longitudinal trial of 26 patients undergoing chemotherapy, 
reported no significant effect of cancer treatment on sleep architecture and sleep continuity. ${ }^{33}$ Interestingly, studies have generally found at best a moderate relationship between subjective and objective measurements of sleep. ${ }^{11-13}$

The use of different sleep questionnaires, methods (eg, actigraphy), and various cutoff points for sleep problems has produced a wide range in the reported prevalence of sleep problems among cancer patients. In addition, differences in terminology and definitions of sleep problems further complicate the issue. Efforts should be made to use precise terminology when describing sleep problems in cancer patients. In scientific literature involving cancer patients, commonly, insomnia, sleep disturbance, poor sleep quality, and insufficient sleep duration are terms used interchangeably. The most commonly used validated patientreported questionnaires for sleep, ideally in association with objective measures, should be utilized in future research to provide reliable estimates of cancer-related sleep problems, to allow comparisons across studies and to elucidate the effect of interventions targeting sleep problems.

\section{Etiological factors for sleep problems in cancer patients}

In part because of the variability in methods for assessing the prevalence of sleep problems in cancer patients and the multifaceted nature of sleep problems both in general and in cancer patients specifically, ${ }^{8,34-36}$ the etiology and predictors of sleep problems in cancer are not entirely understood. The Behavioral Model $^{37}$ proposed by Spielman et al depicts how sleep problems might develop and become chronic. This model suggests that sleep problems occur as a result of predisposing and precipitating factors and may become chronic as a result of perpetuating factors (see Figure 1). Predisposing factors include trait anxiety, predisposition to rumination, and sleep chronotype (late types are more likely to have sleep problems). ${ }^{38}$ Precipitating factors include acute triggers such as stressful or traumatic life events, including cancer diagnosis, concomitant medical illness, or mental health disturbances. Perpetuating factors are behaviors that people develop to compensate for sleep loss. These behaviors are initially adaptive, particularly during sickness, but later contribute to the development of chronic sleep problems. They include napping, spending prolonged time in bed without sleeping, and going to bed earlier and/or getting out of bed later. They lead to extended sleep opportunity and dysregulation of sleep-wake cycles, making it difficult for individuals to fall and stay asleep during regular sleep hours. Thus, this model posits that sleep problems might be maintained via perpetuating factors even when the original stressor (precipitating factor) is no longer present. In addition to focusing on maladaptive

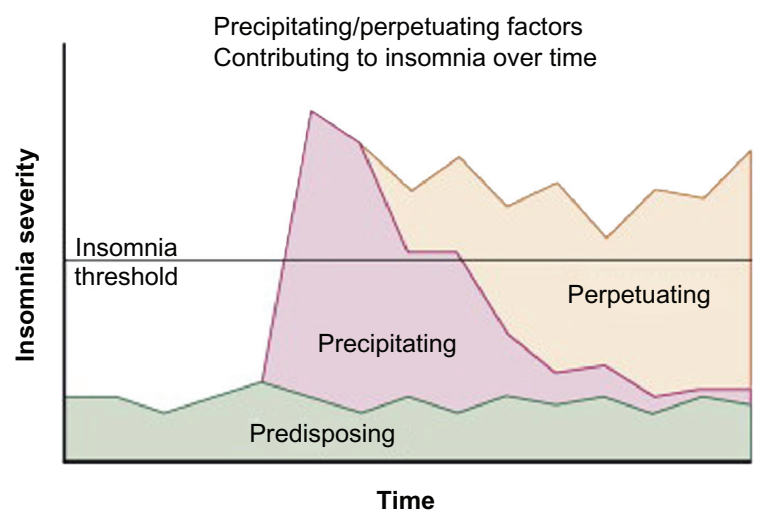

Figure I Conceptual model of the development of chronic insomnia and the changing factors that play a role over the course of the disorder.

Notes: In this particular case, at the onset of the insomnia, precipitating factors predominate. When the insomnia becomes chronic, perpetuating factors become the main feature contributing to the sleep disturbance.

Reprinted from Principles and Practices of Sleep Medicine, 5th ed. In: Spielman, Yang, Glovinsky, editors. Chapter I44-Assessment Techniques for Insomnia; Pages 1632-1645, adapted from Spielman, Caruso, Glovinsky. Copyright Elsevier 2011. Reprinted with permission from Elsevier. ${ }^{151}$

behaviors, cognitive behavioral therapy for insomnia (CBT-I) recommends teaching patients cognitive restructuring and addresses catastrophic thinking associated with having sleep problems and erroneous beliefs about catastrophic consequences (eg, I will get sick, I will be awake the whole night, I will have more side effects associated with cancer treatments). ${ }^{39,40}$

In cancer patients, the precipitating factors for the development of sleep problems are plentiful and include the diagnosis of cancer, the severity of the disease (stage and molecular phenotype), side effects associated with treatments (eg, fatigue, nausea/vomiting, nocturia, hot flashes, itching, and diarrhea), and physical and mental health comorbidities (eg, pain, dyspnea, depression, and anxiety). ${ }^{41}$ Cytotoxic antineoplastic agents often cause adverse effects potentially impairing sleep, at least in a transient way with no clear class-specificity evident. ${ }^{31,42}$ Acute transient but profound circadian disruption induced by chemotherapy has further been demonstrated in two studies involving locomotor activity and temperature monitoring in advanced cancer patients receiving treatment. ${ }^{43,44}$ Indeed, sleep is a circadian phenomenon and dysregulation of circadian rhythms may be a carcinogen that induces and promotes cancer and its growth in laboratory animals and cancer patients. ${ }^{45-53}$

\section{Biological mechanisms contributing to chemotherapy-associated sleep problems Inflammation}

Substantial evidence supports a strong bidirectional relationship between immune functioning and sleep: sleep deprivation 
lowers immune responses, and acute infection, with its subsequent inflammatory response, changes sleep architecture by increasing slow-wave sleep and reducing rapid eye movement sleep. ${ }^{54}$ A complex network of critical immune signaling molecules, specifically cytokines and their receptors, influences sleep mechanisms in the brain through autocrine and paracrine pathways that can have direct or indirect effects. Cytokines are proteins that promote growth, activation, and differentiation of immune cells and affect numerous other cell types. Cytokines can promote inflammation, dampen the immune response, or suppress immunity depending on the type of immune cells and their responsiveness to the cytokines, and they can induce, maintain, or disrupt sleep (for review see Kapsimalis et $\mathrm{al}^{55}$ ). For example, IL-1 $\beta$, TNF- $\alpha$, and IL-6 are important cytokine inducers of sleep, while IL-10 and TNF- $\alpha$ receptor are known inhibitors of sleep. ${ }^{55}$

Studies suggest that pro-inflammatory cytokines are possible mediators of chemotherapy-induced systemic symptoms and associated sleep-wake disturbances. ${ }^{56-60}$ Inflammation resulting from cancer treatments can lead to sleep problems and, conversely, dysregulation of sleep can lead to altered immune profiles, making chronic inflammatory processes a predictor of acute and long-term poor sleep quality in cancer patients and survivors. Immune function is also tightly connected to neuroendocrine function and circadian rhythm; thus, imbalance in these systems can lead to sleep problems..$^{59,61,62}$ Moreover, high concentrations of circulating proinflammatory cytokines such as IL-6, TNF- $\alpha$, and TGF- $\alpha$ have been shown to be associated with disrupted circadian function in patients with advanced cancer. ${ }^{63}$

Furthermore, preliminary studies to date have demonstrated associations between increased pro-inflammatory cytokine levels and sleep impairment in cancer patients during chemotherapy and in cancer survivors ${ }^{64,65}$ Miaskowski et al demonstrated that an IL-6 polymorphism was associated with sleep disturbance in cancer patients receiving radiation therapy. ${ }^{66}$ Sprod et al showed that, in breast and prostate cancer patients, increased IL-6 was associated with reduced sleep efficiency, reduced sleep duration, and increased sleep medication usage ${ }^{67}$ Additionally, in the same study, increased TNF- $\alpha$ was associated with increased sleep latency and increased sleep medication usage. ${ }^{67}$

\section{Circadian disruption}

Studies by Savard et $\mathrm{al}^{31}$ and Palesh et $\mathrm{al}^{68}$ have shown that flattened diurnal cortisol slopes and disrupted circadian rhythms are associated with sleep disturbance or frequent awakenings in cancer patients during chemotherapy and other cancer treatments. Indeed, the circadian component of sleep homeostasis can be profoundly disrupted in cancer patients, as shown by flattened patterns of salivary cortisol or by altered locomotor activity rhythm in patients with advanced cancers. ${ }^{48}$ Furthermore, disrupted circadian rhythms blunt physiological synchronizers of the biological clock such as light exposure, social life, physical activity, and feeding schedule, thus increasing daytime dysfunction due to poor nighttime rest. ${ }^{48,69-71}$

\section{Pharmaceutical agents}

Certain drugs might be particularly disruptive for sleep. For example, immunomodulatory agents used against cancer, such as IFN- $\alpha 2 b$ and IL-2, have been shown to cause depressive symptoms in cancer patients. ${ }^{72}$ This effect could be detrimental for sleep in patients undergoing treatment with these agents, since depression and sleep problems commonly co-occur and might share common etiology in cancer. ${ }^{15}$ Synthetic glucocorticoids administered at therapeutic doses, used either as anticancer agents or for supportive care (used for their antiemetic or immunosuppressive properties), can disrupt physiological diurnal cortisol rhythms, thus altering the circadian component of sleep. ${ }^{59,73}$ Finally, newer targeted agents, such as small tyrosine-kinase inhibitors, could act specifically in the central nervous system, modifying the effect of neuroendocrine cytokines and growth factors on sleep-wake and rest-activity cycles. ${ }^{63,74}$ Gefitinib, for example, an oral epidermal growth factor receptor (EGFR) inhibitor, has been shown to improve circadian rest-activity rhythm regardless of its antitumor effects in patients with advanced non-small cell lung cancer. ${ }^{75}$ The supposed mechanism of action likely involves blockade of the EGFRmediated circadian disruption, demonstrated in experimental models. ${ }^{76,77}$

\section{Demographics}

Aging is associated with a significant deterioration of the daily sleep-wake cycle, ${ }^{78}$ especially after the age of 75 years, ${ }^{79}$ and therefore elderly subjects report a higher incidence of sleep problems. ${ }^{80,81}$ It is not clear, however, whether older age is a risk factor for the occurrence of sleep problems in individuals with cancer: older adults are commonly underrepresented in clinical studies. ${ }^{82}$ Moreover, increasing age is associated with the risk of multiple comorbidities, which in themselves could be predisposing, precipitating, or perpetuating risk factors for the development of sleep problems even more so than the cancer itself or its treatments. ${ }^{83}$ Although sleep problems 
are highly prevalent in elderly patients with cancer, ${ }^{84}$ in large studies, older age has rarely been identified as a risk factor for sleep problems and, in fact, younger cancer patients seem to be at higher risk for sleep problems than older cancer patients. ${ }^{7,85}$ It is unclear why younger patients are at risk for the development of sleep problems during treatment, but it is possible that disease severity, more aggressive treatments, and/or expectations for better health among younger patients are responsible for higher sleep problem rates. ${ }^{7}$

In children and adolescents receiving cancer treatment, impaired sleep is more common than in age-matched controls, ${ }^{86-89}$ and it can reach a prevalence of up to $87 \% .{ }^{9,90}$ Sleep problems in pediatric patients with cancer can span the whole spectrum of clinical sleep disorders ${ }^{90}$ and potentially involve environmental, genetic, and drug-related factors. ${ }^{91,92}$ In survivors of childhood cancer, injury to the hypothalamus or the brainstem, treatment-induced endocrine dysfunction, obesity, or history of cranial radiation therapy are known risk factors for the development of sleep complaints. ${ }^{93}$

\section{Other cancer side effects}

Sleep problems rarely occur in isolation and they frequently correlate with other symptoms during chemotherapy including fatigue, anxiety, depression, pain, and cognitive dysfunction. A recent study by Cheng and Yeung ${ }^{3}$ showed that sleep problems, mood disturbance, fatigue, and pain co-occurred in $29 \%$ of the patients receiving chemotherapy or radiation. In women with breast cancer, depressive symptoms and uncontrolled pain have been shown to be predictors of sleep problems. ${ }^{26,94}$ Depression and sleep problems are so linked that sleep problems were significantly reduced with a common antidepressant during chemotherapy, although the effects were small. ${ }^{15}$ Savard and Ivers ${ }^{95}$ recently reported that the administration of chemotherapy or hormone therapy was associated with increased levels of sleep problems. Interestingly, they reported that the negative effect of chemotherapy on sleep problems was significantly mediated by nausea and other digestive symptoms, hot flashes, and pain. ${ }^{95}$

While larger data exist on the association of sleep with depression, fatigue, pain, and anxiety in cancer, the relationships between chemotherapy-related sleep problems and chemotherapy-related cognitive deficits have not been thoroughly examined. Sleep disruption may contribute to the cognitive dysfunction that is often associated with cancer chemotherapy and vice versa. ${ }^{96} \mathrm{Up}$ to $75 \%$ of cancer survivors who have received adjuvant chemotherapy demonstrate significant difficulties with memory, attention, concentration, processing speed, and executive function. ${ }^{97,98}$ The mechanisms underlying these cognitive problems remain largely unclear although similar mechanisms to those involved in sleep disruption have been proposed. ${ }^{97-99}$ Thus, sleep and cognitive problems may share common causative factors that result in an interaction and in a concomitant worsening of these two symptoms. Indeed, sleep deprivation is associated with cognitive impairment in healthy individuals; ${ }^{100}$ thus, poor sleep may also contribute to cognitive difficulties following cancer chemotherapy.

\section{Management of sleep problems in cancer Overview of psychological interventions for sleep problems}

The American Academy of Sleep Medicine recommends psychological and behavioral interventions such as stimulus control therapy, sleep restriction therapy, and relaxation therapy as effective treatments for sleep problems. ${ }^{101}$ These individual treatments are all components of CBT-I, a nonpharmacological approach to treat sleep problems. The conceptual framework of CBT-I is largely based on Spielman's Behavioral Model and specifically targets the perpetuating factors (maladaptive behaviors) that can lead to sleep problems. ${ }^{37}$ Through one or more sessions (typically four to seven) of CBT-I, the therapist suggests changing maladaptive sleep behaviors to new ones that will resynchronize the biological clock and reinforce the association of the bedroom environment with sleep. Cognitive restructuring is also used to challenge the individual's erroneous beliefs about sleep problems and their possible consequences. ${ }^{39,40}$

Studies in both cancer survivors and cancer patients receiving chemotherapy have suggested that administering CBT-I or its variant to participants stabilizes or improves various sleep parameters. ${ }^{102-113}$ Recent randomized controlled trials investigating the efficacy of CBT-I in breast cancer survivors have shown statistically significant improvements in both objective (ie, actigraphy) ${ }^{103-105}$ and subjective (eg, sleep diaries, ISI, and PSQI) sleep measures in groups receiving CBT-I as compared to conventional care. ${ }^{102-110}$ Of note, three out of four randomized controlled trials utilized group therapy rather than individual therapy, suggesting that group therapy is feasible and effective for survivors. ${ }^{103,104,108}$

Studies in cancer survivors outnumber those in cancer patients undergoing treatment, most likely due to feasibility issues. In recent years, only two randomized controlled clinical trials testing the efficacy of CBT-I were conducted specifically in cancer patients receiving chemotherapy. ${ }^{111,114}$ 
Other studies investigated CBT-I in cancer patients undergoing a variety of treatments. ${ }^{112,113}$ Both have also shown results favoring CBT-I over control groups and therefore suggest that sleep problems can be treated during cancer treatment and that interventions can potentially prevent the development of chronic sleep problems after treatment. While studies in breast cancer survivors have often used group therapy as mentioned above, those in cancer patients undergoing treatment have mainly used individualized therapy involving multiple components of CBT-I, some of which were modified to meet the specific needs of cancer patients. Berger et al conducted a randomized controlled trial with 219 breast cancer patients receiving chemotherapy. ${ }^{111}$ The intervention used was an Individualized Sleep Promotion Plan (ISPP) that patients composed before initiation of chemotherapy with the guidance of research nurses and then revised and reinforced throughout chemotherapy treatment. The ISPP included stimulus control, modified sleep restriction, relaxation therapy, and sleep hygiene. Sleep restriction techniques were modified to allow one additional hour of sleep if the patient could not get up on time and up to two naps per day if the patient felt overly fatigued. Over a period of one year after the first chemotherapy treatment, the treatment group, on average, showed significant improvement in global sleep quality when compared to the healthy eating control group, thereby supporting the use of a modified version of CBT-I as an effective intervention for sleep problems among cancer patients. Furthermore, Dalton et al compared profile-tailored CBT-I, standard CBT-I, and usual care in their study determining the effectiveness of CBT-I for cancer pain and showed that profile-tailored CBT-I patients experienced less interference of pain with sleep than standard CBT-I patients. ${ }^{12}$ Although it focused mainly on cancer pain, Dalton's study lends further support to the efficacy of tailoring CBT-I to meet the individual needs of cancer patients undergoing treatment.

Studies in both cancer patients and survivors have differed in determining who delivers the therapy to the patients. Some of those who conducted the therapy were psychologists and others were registered nurses. Studies in cancer patients undergoing chemotherapy have most often utilized therapy delivered by nurses. Arving et al compared nurse-delivered and psychologist-delivered CBT-I in breast cancer patients undergoing chemotherapy and found that the group who received the therapy from nurses showed the greatest improvement in insomnia. ${ }^{114}$ Patients in this group also reported higher levels of perceived benefit. Although nurses may not be the most qualified to deliver CBT-I, in most cases, the authors suggested that nurses may be a more realistic choice in routine cancer care due to the limited number of psychologists working in cancer centers. More research is needed to investigate this aspect of the intervention to help determine conditions for optimal intervention delivery in the cancer patient population.

Lastly, while therapeutic effects have been found to be sustained for up to 12 months in cancer survivors in some studies, ${ }^{103,115}$ there have been conflicting data on long-term benefits of cognitive behavioral therapy when administered during chemotherapy. The study by Berger et al showed more significant improvement in insomnia in the intervention group than in the control group (healthy eating) 1 year after treatment. ${ }^{111}$ Conversely, the study by Arving et al showed significantly larger improvement in the control (standard care) group than in the intervention group after 6 months. ${ }^{114}$ This inconsistency could be further explored with more data on patient adherence to the new ideas and behaviors taught during the intervention. Berger et al is the only group among recent studies that measured the extent to which participants adhered to the intervention. ${ }^{116}$ They obtained data on self-reported rates of adherence to sleep hygiene counseling (57\%-67\%), stimulus control techniques (46\%-67\%), relaxation therapy (57\%-67\%), and sleep restriction techniques (76\%-79\%). Although the intervention did not improve sleep parameters during chemotherapy, pretreatment sleep parameters remained stable despite the initiation of chemotherapy. Adherence rates remained high for most components after cancer treatment, and sleep-wake patterns stayed within normal limits for up to 1 year after the last chemotherapy treatment, supporting the idea that higher adherence rates could produce longer lasting beneficial effects on sleep. Thus, data on adherence could be valuable in determining the dosage and timing of sessions needed to ensure continued benefits for sleep posttreatment. Nonetheless, in spite of these limitations, recent studies support the notion that behavioral interventions are feasible and effective for reducing sleep problems in cancer patients undergoing chemotherapy.

\section{Overview of pharmacological interventions for sleep problems}

No studies known to the authors have assessed the impact of prescription sleep aids on sleep problems in cancer patients undergoing chemotherapy. Costantini et $\mathrm{al}^{117}$ reported that $32.3 \%$ of cancer patients undergoing chemotherapy were prescribed sleep aids. The most commonly prescribed sleep aids were lorazepam (31.4\%) and zolpidem (29.4\%). This study also found that patients with preexisting psychiatric 
issues and sleep problems were more likely to request and take sleep aids from their provider. These findings were consistent with another study ${ }^{118}$ that examined the use of sleep aids during breast cancer adjuvant chemotherapy in 219 patients. That study reported that approximately $20 \%$ of cancer patients took at least one sleep medication before chemotherapy. Use of sleep aids decreased over time from $11 \%$ to $6 \%$ as chemotherapy treatment progressed. The majority of sleep aids (46\%) used by the patients were prescription sedatives/hypnotics (eg, zolpidem, lorazepam) followed by $24 \%$ of patients taking analgesics (eg, ibuprofen). ${ }^{118}$ Altogether, these findings show that a significant minority of patients diagnosed with cancer use sleep aids. The effects of using sleep aid medications during chemotherapy treatment are unknown, but their use can lead to potentially harmful pharmacological interactions. ${ }^{119}$ However, no randomized clinical trial to date has evaluated the efficacy or the safety of hypnotics in cancer patients on chemotherapy.

\section{Overview of exercise for sleep problems}

Research suggests that exercise can improve sleep problems in the general population. ${ }^{120}$ However, the mode, frequency, duration, intensity, and daily timing of exercise and the current fitness level of the individual all significantly impact the degree of efficacy of exercise on sleep. ${ }^{120}$ Exercise regimens that incorporate aerobic and/or anaerobic modes of physical activity performed very frequently (eg, daily or multiple times a day), for extended periods of time, at high intensity resulting in overtraining can lead to poor overall sleep quality and shortened sleep time. ${ }^{121}$ Research suggests that exercise performed early during the day improves sleep, whereas results are mixed regarding exercise performed later during the day. ${ }^{120}$ Individuals who already exercise regularly will not experience the same magnitude of sleep improvements as individuals who are sedentary and begin a regular exercise program. ${ }^{120,122}$ Traditional forms of physical exercise, such as walking and resistance exercise, are safe and provide many benefits for cancer patients and survivors including improvements in sleep. ${ }^{123-126}$

\section{Exercise for sleep problems throughout the cancer trajectory}

\section{Aerobic walking programs}

Breast cancer survivors receiving hormonal treatment demonstrated improvements in sleep quality following a 12-week moderate level walking intervention (4 days a week, 20 minutes per day). ${ }^{127}$ Tang et al also showed that brisk walking ( 3 days a week, 30 minutes a day for 8 weeks) resulted in improvements in sleep quality and in quality of life in cancer patients. ${ }^{129}$ Mock et al assessed the effects of a home-based walking exercise intervention (4-5 days a week, 20-30 minutes a day for 6 weeks) on a variety of outcomes, including sleep and cancer-related fatigue in breast cancer patients who were undergoing radiation treatments. In this non-randomized study, the home-based exercise intervention resulted in improved sleep outcomes, such as difficulty sleeping, as well as physical functioning, fatigue, and anxiety. ${ }^{129}$ Wang et al also showed that a low to moderate intensity walking program (3-5 days a week, 30-50 minutes a day for 6 weeks) improved sleep disturbance in women with stage I or II breast cancer. ${ }^{130}$

\section{Aerobic training combined with anaerobic resistance training programs}

A 4-week, low to moderate intensity home-based walking and resistance-band exercise intervention (7 days a week for 4 weeks) resulted in improved sleep quality in breast and prostate cancer patients undergoing radiation treatments. ${ }^{67}$ Additionally, a study by Young-McCaughan et al, used a Phase II cardiac rehabilitation model of 12 weeks of individualized supervised exercise and education in cancer patients, and the model was found to have beneficial effects on self-reported sleep. ${ }^{131}$ Coleman et al conducted a small, randomized, controlled trial including 24 patients receiving high-dose chemotherapy and autologous peripheral blood stem cell transplantation for multiple myeloma. Patients randomized to the home-based exercise intervention, which consisted of both personalized aerobic and resistance exercise (varying in intensity, duration, and frequency of exercise) reported a nonsignificant trend towards increased total minutes of sleep and sleep efficiency. The intervention did not result in any adverse events. ${ }^{132}$ In another study, 119 women with breast, colon, or ovarian cancer were randomized into three groups: to receive a home-based exercise intervention (3-5 times a week, 20-30 minutes a day, at $60 \%-80 \% \mathrm{VO}_{2}$ peak) during chemotherapy; to receive the same home-based exercise intervention following treatment completion; or to usual care. The authors found no significant improvements in sleep in the intervention groups as compared to standard care. ${ }^{133}$

\section{Mindfulness exercise training programs}

Yoga is an increasingly popular alternative mode of physical exercise in the United States and other countries as well. ${ }^{134,135}$ There are many different types of yoga ranging from styles that focus predominantly on meditation and breathing with no physical activity to styles that incorporate meditation, 
breathing, and vigorous physical activity and styles that are taught in hot rooms $\left(\mathrm{eg}, \geq 95^{\circ} \mathrm{F} / 35^{\circ} \mathrm{C}\right)$. Evidence suggests that different types of yoga are safe and may improve sleep among cancer patients and survivors. ${ }^{136-140}$ Recently, Mustian et al completed the largest and most definitive clinical trial of yoga in cancer patients to date, demonstrating that yoga improves sleep. ${ }^{125}$ The intervention used the standardized Yoga for Cancer Survivors (YOCAS ${ }^{\circledR}$ ) program, designed by researchers at the University of Rochester Medical Center (Rochester, NY). The YOCAS intervention is based on two forms of yoga: gentle hatha yoga and restorative yoga. The YOCAS sessions are standardized, and each session includes physical alignment postures, breathing, and mindfulness exercises. The intervention is delivered in an instructor-taught group format twice a week for 75 minutes each time over 4 weeks for a total of eight sessions of yoga. ${ }^{125}$

Exercise has shown great promise for treatment of sleep problems in cancer patients and survivors. However, an important limitation of these studies was that the patients usually had non-clinical levels of sleep impairment at baseline. Thus, we cannot be certain that exercise (or yoga) is as effective in patients with clinically significant sleep problems. Dedicated trials are therefore warranted to clarify the efficacy of exercise in cancer patients with clinical sleep problems.

\section{Biological mechanisms associated with exercise and sleep}

Exercise may influence sleep quality through at least two mechanisms: body temperature and proinflammatory cytokines. Sleep induction is mediated by the decrease in core body temperature and increase in superficial temperature, whose patterns can be influenced by exercise. ${ }^{141,142}$ Sleep is also regulated by proinflammatory cytokines. ${ }^{120}$ Regular exercise participation has been shown to reduce low-grade inflammation through its influence on cytokines. ${ }^{143,144}$ Exercise results in the release of IL-6, a cytokine with proand anti-inflammatory properties, from skeletal muscle. ${ }^{145}$ The duration and intensity of exercise and the amount of muscle mass that is used during exercise affects the amount of IL-6 released from skeletal muscle. The release of IL-6 during and immediately following an acute bout of exercise results in the increase of anti-inflammatory molecules such as sTNF-R and in the inhibition in the production of TNF- $\alpha$, which is a proinflammatory cytokine. ${ }^{145-147}$ Through this complex modulation of cytokine release, exercise plays a positive role in sleep quality, especially in those who have impaired sleep.
It is likely that the beneficial effects of exercise on sleep are also mediated by other mechanisms, including an entraining effect on the circadian timing system of the subject (physical activity is an important nonphotic synchronizer in humans), ${ }^{148}$ and an effect on the arousal system, involving the autonomic nervous system tone and the hypothalamus-pituitary-adrenal axis function. ${ }^{149,150}$ Overall, the current knowledge of mechanisms associated with exercise and sleep in cancer is limited, and further research is needed to fully understand the relative implication of each of these central and peripheral mechanisms on the effect of physical exercise on sleep, particularly in patients with cancer undergoing treatment. The acquired data would finally help in the optimization and personalization of exercise as an active and safe nonpharmacological treatment of sleep problems and circadian disruption in cancer patients.

\section{Future directions}

The clinical management of sleep disturbance in patients with cancer receiving chemotherapy needs to be improved. To this end, dedicated research should be performed, using standardized methodology, with the aim of differentiating the relative influence of tumor and cancer treatment on the development and persistence of sleep problems to develop a better understanding of the reciprocal influence of sleep disruption on disease progression, side effects, and toxicities to fully elucidate the implicated mechanisms. This increased knowledge and awareness in this field will thus ultimately lead to the development of optimal and individualized interventions to treat this frequent, multifaceted, and bothersome symptom in patients with cancer.

\section{Summary}

Cancer patients undergoing chemotherapy suffer from a myriad of side effects, including sleep problems. Sleep problems are prevalent and increase in severity during chemotherapy but are undertreated and under-recognized. While the mechanisms behind sleep problems during chemotherapy are not entirely understood, it is clear that chemotherapy dysregulates sleep-wake cycles. Tumor and antitumor treatments both increase the production of proinflammatory cytokines, which in turn act in the central nervous system and alter the rest-activity rhythms and negatively affect sleep. Several evidence-based approaches exist for the management of sleep problems in patients undergoing treatments for their cancer. Modified versions 
of cognitive behavioral interventions have successfully been used in the cancer clinic and have demonstrated efficacy. In addition, exercise, including yoga, has been shown to improve sleep although it is unclear if exercise is potent enough to manage clinically significant sleep problems. No specific pharmacological treatment for sleep problems during chemotherapy has been developed; however, patients use nonprescription and prescription sleep medicine even though the medications haven't been systematically tested in cancer patients. It is important to address sleep problems during cancer treatments because they directly affect patients' abilities to complete treatment for cancer, recover, and ultimately survive with a good quality of life.

\section{Acknowledgment}

This research was supported by NCI K07 CA132916 and NCI K07CA120025. We thank Karyn Haitz for her help with the final editing of this manuscript.

\section{Disclosure}

The authors report no conflicts of interest in this work.

\section{References}

1. Siegel R, Naishadham D, Jemal A. Cancer statistics, 2012. CA Cancer J Clin. 2012;62(1):10-29.

2. Hofman M, Morrow GR, Roscoe JA, et al. Cancer patients' expectations of experiencing treatment-related side effects: a University of Rochester Cancer Center-Community Clinical Oncology Program study of 938 patients from community practices. Cancer. 2004;101(4):851-857.

3. Cheng KK, Yeung RM. Impact of mood disturbance, sleep disturbance, fatigue and pain among patients receiving cancer therapy. Eur J Cancer Care (Engl). Epub July 16, 2012.

4. Davidson JR, MacLean AW, Brundage MD, Schulze K. Sleep disturbance in cancer patients. Soc Sci Med. 2002;54(9):1309-1321.

5. Savard J, Ivers H, Villa J, Caplette-Gingras A, Morin CM. Natural course of insomnia comorbid with cancer: an 18-month longitudinal study. J Clin Oncol. 2011;29(26):3580-3586.

6. Savard J, Villa J, Ivers H, Simard S, Morin CM. Prevalence, natural course, and risk factors of insomnia comorbid with cancer over a 2-month period. J Clin Oncol. 2009;27(31):5233-5239.

7. Palesh OG, Roscoe JA, Mustian KM, et al. Prevalence, demographics, and psychological associations of sleep disruption in patients with cancer: University of Rochester Cancer Center-Community Clinical Oncology Program. J Clin Oncol. 2010;28(2):292-298.

8. Savard J, Morin CM. Insomnia in the context of cancer: a review of a neglected problem. J Clin Oncol. 2001;19(3):895-908.

9. Zupanec S, Jones H, Stremler R. Sleep habits and fatigue of children receiving maintenance chemotherapy for ALL and their parents. $J$ Pediatr Oncol Nurs. 2010;27(4):217-228.

10. Liu L, Fiorentino L, Natarajan L, et al. Pre-treatment symptom cluster in breast cancer patients is associated with worse sleep, fatigue and depression during chemotherapy. Psychooncology. 2009;18(2):187-194.

11. Beck SL, Berger AM, Barsevick AM, Wong B, Stewart KA, Dudley WN Sleep quality after initial chemotherapy for breast cancer. Support Care Cancer. 2010;18(6):679-689.

12. Argyropoulos SV, Hicks JA, Nash JR, Bell CJ, et al. Correlation of subjective and objective sleep measurements at different stages of the treatment of depression. Psychiatry Res. 2003;120(2):179-190.
13. Dhruva A, Paul SM, Cooper BA, et al. A longitudinal study of measures of objective and subjective sleep disturbance in patients with breast cancer before, during, and after radiation therapy. J Pain Symptom Manage. 2012;44(2):215-228.

14. Chen ML, Yu CT, Yang CH. Sleep disturbances and quality of life in lung cancer patients undergoing chemotherapy. Lung Cancer. 2008;62(3):391-400.

15. Palesh OG, Mustian KM, Peppone LJ, et al. Impact of paroxetine on sleep problems in 426 cancer patients receiving chemotherapy: a trial from the University of Rochester Cancer Center Community Clinical Oncology Program. Sleep Med. Epub August 1, 2012.

16. Ancoli-Israel S, Liu L, Marler MR, et al. Fatigue, sleep, and circadian rhythms prior to chemotherapy for breast cancer. Support Care Cancer. 2006;14(3):201-209.

17. Berger AM, Farr LA, Kuhn BR, Fischer P, Agrawal S. Values of sleep/ wake, activity/rest, circadian rhythms, and fatigue prior to adjuvant breast cancer chemotherapy. J Pain Symptom Manage. 2007;33(4): 398-409.

18. Purnell JQ, Mustian K, Jean-Pierre P, et al. The psychosocial and functional impact of radiation therapy. In: Rubin P, Constine LS, Marks LB, et al, editors. ALERT - Adverse Late Effects of Cancer Treatment. Heidelberg: Springer; 2012.

19. Gooneratne NS, Dean GE, Rogers AE, Nkwuo JE, Coyne JC, Kaiser LR. Sleep and quality of life in long-term lung cancer survivors. Lung Cancer. 2007;58(3):403-410.

20. Mulrooney DA, Ness KK, Neglia JP, et al. Fatigue and sleep disturbance in adult survivors of childhood cancer: a report from the Childhood Cancer Survivor Study (CCSS). Sleep. 2008;31(2):271-281.

21. Taylor TR, Huntley ED, Makambi K, et al. Understanding sleep disturbances in African- American breast cancer survivors: a pilot study. Psychooncology. Epub June 5, 2011.

22. Härtl K, Engel J, Herschbach P, Reinecker H, Sommer H, Friese K. Personality traits and psychosocial stress: quality of life over 2 years following breast cancer diagnosis and psychological impact factors Psychooncology. 2010;19(2):160-169.

23. Cooley ME, Short TH, Moriarty HJ. Symptom prevalence, distress, and change over time in adults receiving treatment for lung cancer. Psychooncology. 2003;12(7):694-708.

24. Kenefick AL. Patterns of symptom distress in older women after surgical treatment for breast cancer. Oncol Nurs Forum. 2006;33(2):327-335.

25. Thomas KS, Bower J, Hoyt MA, Sepah S. Disrupted sleep in breast and prostate cancer patients undergoing radiation therapy: the role of coping processes. Psychooncology. 2010;19(7):767-776.

26. Palesh OG, Collie K, Batiuchok D, et al. A longitudinal study of depression, pain, and stress as predictors of sleep disturbance among women with metastatic breast cancer. Biol Psychol. 2007;75(1): 37-44.

27. Berger AM, Farr L. The influence of daytime inactivity and nighttime restlessness on cancer-related fatigue. Oncol Nurs Forum. 1999;26(10): 1663-1671.

28. Roscoe JA, Morrow GR, Hickok JT, et al. Temporal interrelationships among fatigue, circadian rhythm and depression in breast cancer patients undergoing chemotherapy treatment. Support Care Cancer. 2002;10(4):329-336.

29. Carpenter JS, Andrykowski MA. Psychometric evaluation of the Pittsburgh Sleep Quality Index. J Psychosom Res. 1998;45(1):5-13.

30. Savard MH, Savard J, Simard S, Ivers H. Empirical validation of the Insomnia Severity Index in cancer patients. Psychooncology. 2005;14(6):429-441.

31. Savard J, Liu L, Natarajan L, et al. Breast cancer patients have progressively impaired sleep-wake activity rhythms during chemotherapy. Sleep. 2009;32(9):1155-1160.

32. Parker KP, Bliwise DL, Ribeiro M, et al. Sleep/Wake patterns of individuals with advanced cancer measured by ambulatory polysomnography. J Clin Oncol. 2008;26(15):2464-2472.

33. Roscoe JA, Perlis ML, Pigeon WR, et al. Few changes observed in polysomnographic-assessed sleep before and after completion of chemotherapy. J Psychosom Res. 2011;71(6):423-428. 
34. Pack AI, Pien GW. Update on sleep and its disorders. Annu Rev Med. 2011;62:447-460.

35. Vgontzas AN, Kales A. Sleep and its disorders. Annu Rev Med. 1999;50: $387-400$.

36. Berger AM. Update on the state of the science: sleep-wake disturbances in adult patients with cancer. Oncol Nurs Forum. 2009;36(4): E165-E177.

37. Spielman AJ, Caruso LS, Glovinsky PB. A behavioral perspective on insomnia treatment. Psychiatr Clin North Am. 1987;10(4):541-553.

38. Ong JC, Huang JS, Kuo TF, Manber R. Characteristics of insomniacs with self-reported morning and evening chronotypes. J Clin Sleep Med. 2007;3(3):289-294.

39. Perlis M, Jungquist C, Smith M, Posner D. Cognitive behavioral treatment of insomnia: A session-by-session guide. New York: Springer; 2005.

40. Edinger J, Carney C. Overcoming insomnia. A cognitive-behavioral therapy approach. New York: Oxford University Press; 2008.

41. Ancoli-Israel S. Recognition and treatment of sleep disturbances in cancer. J Clin Oncol. 2009;27(35):5864-5866.

42. Yamagishi A, Morita T, Miyashita M, Kimura F. Symptom prevalence and longitudinal follow-up in cancer outpatients receiving chemotherapy. J Pain Symptom Manage. 2009;37(5):823-830.

43. Ortiz-Tudela EIP, Rol MA, Madrid JA, Lévi F. Circadian patterns in integrated wrist temperature, rest-activity, and position (TAP) as a biomarker for personalized cancer chronotherapeutics. SRBR Meeting. 2012 May 19-23; Florida, USA.

44. Ortiz-Tudela EIP, Iurisci I, Karaboué A, et al. Chemotherapy-induced circadian disruption in cancer patients. XII Congress of the European Biological Rhythms Society; August 20-26, 2011; Oxford, UK.

45. Filipski E, Lévi F. Circadian disruption in experimental cancer processes. Integr Cancer Ther. 2009;8(4):298-302.

46. Straif K, Baan R, Grosse Y, et al. Carcinogenicity of shift-work, painting, and fire-fighting. Lancet Oncol. 2007;8(12):1065-1066.

47. Filipski E, Innominato PF, Wu M, et al. Effects of light and food schedules on liver and tumor molecular clocks in mice. J Natl Cancer Inst. 2005;97(7):507-517.

48. Innominato PF, Focan $\mathrm{C}$, Gorlia $\mathrm{T}$, et al. Circadian rhythm in rest and activity: a biological correlate of quality of life and a predictor of survival in patients with metastatic colorectal cancer. Cancer Res. 2009;69(11):4700-4707.

49. Innominato PF, Giacchetti S, Bjarnason GA, et al. Prediction of overall survival through circadian rest-activity monitoring during chemotherapy for metastatic colorectal cancer. International journal of cancer. Int $J$ Cancer. 2012;131(11):2684-2692.

50. Filipski E, Delaunay F, King VM, et al. Effects of chronic jet lag on tumor progression in mice. Cancer Res. 2004;64(21):7879-7885.

51. Filipski E, King VM, Li X, et al. Host circadian clock as a control point in tumor progression. J Natl Cancer Inst. 2002;94(9): 690-697.

52. Mormont MC, Waterhouse J, Bleuzen P, et al. Marked 24-h rest/ activity rhythms are associated with better quality of life, better response, and longer survival in patients with metastatic colorectal cancer and good performance status. Clin Cancer Res. 2000;6(8): 3038-3045.

53. Sephton SE, Sapolsky RM, Kraemer HC, Spiegel D. Diurnal Cortisol Rhythm as a Predictor of Breast Cancer Survival. J Natl Cancer Inst. 2000;92(12):994-1000.

54. Majde JA, Krueger JM. Links between the innate immune system and sleep. J Allergy Clin Immunol. 2005;116(6):1188-1198.

55. Kapsimalis F, Richardson G, Opp MR, Kryger M. Cytokines and normal sleep. Curr Opin Pulm Med. 2005;11(6):481-484.

56. Dantzer R, O’Connor JC, Freund GG, Johnson RW, Kelley KW. From inflammation to sickness and depression: when the immune system subjugates the brain. Nat Rev Neurosci. 2008;9(1):46-56.

57. Reyes-Gibby CC, Wu X, Spitz M, et al. Molecular epidemiology, cancer-related symptoms, and cytokines pathway. Lancet Oncol. 2008;9(8):777-785.
58. Seruga B, Zhang H, Bernstein LJ, Tannock IF. Cytokines and their relationship to the symptoms and outcome of cancer. Nat Rev Cancer. 2008;8(11):887-899.

59. Miller AH, Ancoli-Israel S, Bower JE, Capuron L, Irwin MR. Neuroendocrine-immune mechanisms of behavioral comorbidities in patients with cancer. J Clin Oncol. 2008;26(6):971-982.

60. Mills PJ, Ancoli-Israel S, Parker B, et al. Predictors of inflammation in response to anthracycline-based chemotherapy for breast cancer. Brain Behav Immun. 2008;22(1):98-104.

61. Lee BN, Dantzer R, Langley KE, et al. A cytokine-based neuroimmunologic mechanism of cancer-related symptoms. Neuroimmunomodulation. 2004;11(5):279-292.

62. Dantzer R, Kelley KW. Twenty years of research on cytokine-induced sickness behavior. Brain Behav Immun. 2007;21(2):153-160.

63. Rich T, Innominato PF, Boerner J, et al. Elevated serum cytokines correlated with altered behavior, serum cortisol rhythm, and dampened 24-hour rest-activity patterns in patients with metastatic colorectal cancer. Clin Cancer Res. 2005;11(5):1757-1764.

64. Wang XS, Shi Q, Williams LA, et al. Inflammatory cytokines are associated with the development of symptom burden in patients with NSCLC undergoing concurrent chemoradiation therapy. Brain Behav Immun. 2010;24(6):968-974.

65. Clevenger L, Schrepf A, Christensen D, et al. Sleep disturbance, cytokines, and fatigue in women with ovarian cancer. Brain Behav Immun. 2012;26(7):1037-1044.

66. Miaskowski C, Dodd M, Lee K, et al. Preliminary evidence of an association between a functional interleukin-6 polymorphism and fatigue and sleep disturbance in oncology patients and their family caregivers. J Pain Symptom Manage. 2010;40(4):531-544.

67. Sprod LK, Palesh OG, Janelsins MC, et al. Exercise, sleep quality, and mediators of sleep in breast and prostate cancer patients receiving radiation therapy. Community Oncol. 2010;7(10):463-471.

68. Palesh O, Zeitzer JM, Conrad A, et al. Vagal regulation, cortisol, and sleep disruption in women with metastatic breast cancer. J Clin Sleep Med. 2008;4(5):441-449.

69. Ancoli-Israel S, Rissling M, Neikrug A, et al. Light treatment prevents fatigue in women undergoing chemotherapy for breast cancer. Support Care Cancer. 2012;20(6):1211-1219.

70. Liu L, Marler MR, Parker BA, et al. The relationship between fatigue and light exposure during chemotherapy. Support Care Cancer. 2005; 13(12):1010-1017.

71. Lévi F, Okyar A, Dulong S, Innominato PF, Clairambault J. Circadian timing in cancer treatments. Annu Rev Pharmacol Toxicol. 2010;50: $377-421$.

72. Capuron L, Ravaud A, Dantzer R. Early depressive symptoms in cancer patients receiving interleukin 2 and/or interferon alfa-2b therapy. J Clin Oncol. 2000;18(10):2143-2151.

73. Innominato PF, Levi FA, Bjarnason GA. Chronotherapy and the molecular clock: Clinical implications in oncology. Adv Drug Deliv Rev. 2010;62(9-10):979-1001.

74. Eismann EA, Lush E, Sephton SE. Circadian effects in cancer-relevant psychoneuroendocrine and immune pathways. Psychoneuroendocrinology. 2010;35(7):963-976.

75. Lurisci I, Rich T, Lévi F, et al. Relief of symptoms after gefitinib is associated with improvement of rest/activity rhythm in advanced lung cancer. J Clin Oncol. 2007;25(16):e17-e19.

76. Kramer A, Yang FC, Snodgrass P, et al. Regulation of daily locomotor activity and sleep by hypothalamic EGF receptor signaling. Science. 2001;294(5551):2511-2515.

77. Snodgrass-Belt P, Gilbert JL, Davis FC. Central administration of transforming growth factor-alpha and neuregulin-1 suppress active behaviors and cause weight loss in hamsters. Brain Res. 2005;1038(2): 171-182.

78. Crowley K. Sleep and sleep disorders in older adults. Neuropsychol Rev. 2011;21(1):41-53.

79. Asplund R. Sleep disorders in the elderly. Drugs Aging. 1999;14(2): 91-103. 
80. Foley DJ, Monjan A, Simonsick EM, Wallace RB, Blazer DG. Incidence and remission of insomnia among elderly adults: an epidemiologic study of 6,800 persons over three years. Sleep. 1999;22 Suppl 2: S366-S372.

81. Ohayon MM, Carskadon MA, Guilleminault C, Vitiello MV. Metaanalysis of quantitative sleep parameters from childhood to old age in healthy individuals: developing normative sleep values across the human lifespan. Sleep. 2004;27(7):1255-1273.

82. Townsley CA, Selby R, Siu LL. Systematic review of barriers to the recruitment of older patients with cancer onto clinical trials. J Clin Oncol. 2005;23(13):3112-3124.

83. Patnaik JL, Byers T, Diguiseppi C, Denberg TD, Dabelea D. The influence of comorbidities on overall survival among older women diagnosed with breast cancer. J Natl Cancer Inst. 2011;103(14):1101-1111.

84. Cheng KK, Lee DT. Effects of pain, fatigue, insomnia, and mood disturbance on functional status and quality of life of elderly patients with cancer. Crit Rev Oncol Hematol. 2011;78(2):127-137.

85. Mohile SG, Heckler C, Fan L, et al. Age-related Differences in Symptoms and Their Interference with Quality of Life in 903 Cancer Patients Undergoing Radiation Therapy. J Geriatr Oncol. 2011;2(4): 225-232.

86. van Litsenburg RR, Huisman J, Hoogerbrugge PM, Egeler RM, Kaspers GJ, Gemke RJ. Impaired sleep affects quality of life in children during maintenance treatment for acute lymphoblastic leukemia: an exploratory study. Health Qual Life Outcomes. 2011;9:25.

87. Wright M. Children receiving treatment for cancer and their caregivers: a mixed methods study of their sleep characteristics. Pediatr Blood Cancer. 2011;56(4):638-645.

88. Erickson JM, Beck SL, Christian BR, et al. Fatigue, sleep-wake disturbances, and quality of life in adolescents receiving chemotherapy. J Pediatr Hematol Oncol. 2011;33(1):e17-e25.

89. Walker AJ, Johnson KP, Miaskowski C, Lee KA, Gedaly-Duff V. Sleep quality and sleep hygiene behaviors of adolescents during chemotherapy. Journal of clinical sleep medicine. J Clin Sleep Med 2010;6(5):439-444.

90. Rosen G, Brand SR. Sleep in children with cancer: case review of 70 children evaluated in a comprehensive pediatric sleep center. Support Care Cancer. 2011;19(7):985-994.

91. Vallance K, Yang J, Li J, Crabtree VM, Hinds PS, Mandrell BN. Disturbed sleep in pediatric patients with leukemia: the potential role of interleukin-6 (-174GC) and tumor necrosis factor (-308GA) polymorphism. Oncol Nurs Forum. 2011;38(5):E365-E372.

92. Hinds PS, Hockenberry MJ, Gattuso JS, et al. Dexamethasone alters sleep and fatigue in pediatric patients with acute lymphoblastic leukemia. Cancer. 2007;110(10):2321-2330.

93. Rosen GM, Shor AC, Geller TJ. Sleep in children with cancer. Curr Opin Pediatr. 2008;20(6):676-681.

94. Koopman C, Nouriani B, Erickson V, et al. Sleep disturbances in women with metastatic breast cancer. Breast J. 2002;8(6):362-370.

95. Savard J, Ivers H. The initiation of chemotherapy, but not radiation therapy, coincides with increased insomnia. Psychooncology. 2012;21: 37-38.

96. Caplette-Gingras A, Savard J, Savard MH, Ivers H. Is Insomnia Associated With Cognitive Impairments in Breast Cancer Patients? Behav Sleep Med. 2012. [Epub ahead of print] PubMed PMID:23181706.

97. Janelsins MC, Kohli S, Mohile SG, Usuki K, Ahles TA, Morrow GR. An update on cancer- and chemotherapy-related cognitive dysfunction: current status. Semin Oncol. 2011;38(3):431-438.

98. Wefel JS, Vardy J, Ahles T, Schagen SB. International Cognition and Cancer Task Force recommendations to harmonise studies of cognitive function in patients with cancer. Lancet Oncol. 2011;12(7): 703-708.

99. Ahles TA, Saykin AJ. Candidate mechanisms for chemotherapyinduced cognitive changes. Nat Rev Cancer. 2007;7(3):192-201.

100. Rakitin BC, Tucker AM, Basner RC, Stern Y. The effects of stimulus degradation after 48 hours of total sleep deprivation. Sleep. 2012;35(1): 113-121.
101. Morgenthaler T, Kramer M, Alessi C, et al; for American Academy of Sleep Medicine. Practice parameters for the psychological and behavioral treatment of insomnia: an update. An american academy of sleep medicine report. Sleep. 2006;29(11):1415-1419.

102. Davidson JR, Waisberg JL, Brundage MD, MacLean AW. Nonpharmacologic group treatment of insomnia: a preliminary study with cancer survivors. Psychooncology. 2001;10(5):389-397.

103. Espie CA, Fleming L, Cassidy J, et al. Randomized controlled clinical effectiveness trial of cognitive behavior therapy compared with treatment as usual for persistent insomnia in patients with cancer J Clin Oncol. 2008;26(28):4651-4658

104. Epstein DR, Dirksen SR. Randomized trial of a cognitive-behavioral intervention for insomnia in breast cancer survivors. Oncol Nurs Forum. 2007;34(5):E51-E59.

105. Fiorentino L, McQuaid JR, Liu L, et al. Individual cognitive behavioral therapy for insomnia in breast cancer survivors: a randomized controlled crossover pilot study. Nat Sci Sleep. 2009;2010:1-8.

106. Hunter MS, Coventry S, Hamed H, Fentiman I, Grunfeld EA. Evaluation of a group cognitive behavioural intervention for women suffering from menopausal symptoms following breast cancer treatment. Psychooncology. 2009;18(5):560-563.

107. Quesnel C, Savard J, Simard S, Ivers H, Morin CM. Efficacy of cognitive-behavioral therapy for insomnia in women treated for nonmetastatic breast cancer. J Consult Clin Psychol. 2003;71(1) 189-200.

108. Savard J, Simard S, Ivers H, Morin CM. Randomized study on the efficacy of cognitive-behavioral therapy for insomnia secondary to breast cancer, part II: Immunologic effects. J Clin Oncol. 2005;23(25): 6097-6106.

109. Savard J, Villa J, Simard S, Ivers H, Morin CM. Feasibility of a selfhelp treatment for insomnia comorbid with cancer. Psychooncology. 2011;20(9):1013-1019.

110. Simeit R, Deck R, Conta-Marx B. Sleep management training for cancer patients with insomnia. Support Care Cancer. 2004;12(3):176-183.

111. Berger AM, Kuhn BR, Farr LA, et al. One-year outcomes of a behavioral therapy intervention trial on sleep quality and cancer-related fatigue. J Clin Oncol. 2009;27(35):6033-6040.

112. Dalton JA, Keefe FJ, Carlson J, Youngblood R. Tailoring cognitivebehavioral treatment for cancer pain. Pain Manag Nurs. 2004;5(1) 3-18.

113. Savard J, Simard S, Giguère I, et al. Randomized clinical trial on cognitive therapy for depression in women with metastatic breast cancer: psychological and immunological effects. Palliat Support Care. 2006;4(3):219-237.

114. Arving C, Sjödén PO, Bergh J, et al. Individual psychosocial support for breast cancer patients: a randomized study of nurse versus psychologist interventions and standard care. Cancer Nurs. 2007;30(3): E10-E19.

115. Savard J, Simard S, Ivers H, Morin CM. Randomized study on the efficacy of cognitive-behavioral therapy for insomnia secondary to breast cancer, part I: Sleep and psychological effects. J Clin Oncol. 2005;23(25):6083-6096.

116. Berger AM, VonEssen S, Kuhn BR, et al. Adherence, sleep, and fatigue outcomes after adjuvant breast cancer chemotherapy: results of a feasibility intervention study. Oncol Nurs Forum. 2003;30(3):513-522.

117. Costantini C, Ale-Ali A, Helsten T. Sleep aid prescribing practices during neoadjuvant or adjuvant chemotherapy for breast cancer. J Palliat Med. 2011;14(5):563-566.

118. Moore TA, Berger AM, Dizona P. Sleep aid use during and following breast cancer adjuvant chemotherapy. Psychooncology. 2011;20(3): 321-325.

119. Riechelmann RP, Tannock IF, Wang L, Saad ED, Taback NA, Krzyzanowska MK. Potential drug interactions and duplicate prescriptions among cancer patients. J Natl Cancer Inst. 2007;99(8): 592-600.

120. Driver HS, Taylor SR. Exercise and sleep. Sleep Med Rev. 2000;4(4): $387-402$. 
121. Smith LL. Cytokine hypothesis of overtraining: a physiological adaptation to excessive stress? Med Sci Sports Exerc. 2000;32(2): 317-331.

122. Youngstedt SD, O'Connor PJ, Dishman RK. The effects of acute exercise on sleep: a quantitative synthesis. Sleep. 1997;20(3):203-214.

123. Mustian KM, Griggs JJ, Morrow GR, et al. Exercise and side effects among 749 patients during and after treatment for cancer: a University of Rochester Cancer Center Community Clinical Oncology Program Study. Support Care Cancer. 2006;14(7):732-741.

124. Mustian KM, Morrow GR, Carroll JK, Figueroa-Moseley CD, Jean-Pierre P, Williams GC. Integrative nonpharmacologic behavioral interventions for the management of cancer-related fatigue. Oncologist. 2007;12 Suppl 1:52-67.

125. Mustian KM, Palesh OG, Sprod LK, et al. Effect of YOCAS yoga on sleep, fatigue, and quality and life: A URCC CCOP randomized, controlled clinical trial among 410 cancer survivors. J Clin Oncol. 2010;28:(15s):9013.

126. Mustian KM, Sprod LK, Palesh OG, et al. Exercise for the management of side effects and quality of life among cancer survivors. Curr Sports Med Rep. 2009;8(6):325-330.

127. Payne JK, Held J, Thorpe J, Shaw H. Effect of exercise on biomarkers, fatigue, sleep disturbances, and depressive symptoms in older women with breast cancer receiving hormonal therapy. Oncol Nurs Forum. 2008;35(4):635-642.

128. Tang MF, Liou TH, Lin CC. Improving sleep quality for cancer patients: benefits of a home-based exercise intervention. Support Care Cancer. 2010;18(10):1329-1339.

129. Mock V, Dow KH, Meares CJ, et al. Effects of exercise on fatigue, physical functioning, and emotional distress during radiation therapy for breast cancer. Oncol Nurs Forum. 1997;24(6):991-1000.

130. Wang YJ, Boehmke M, Wu YW, Dickerson SS, Fisher N. Effects of a 6-week walking program on Taiwanese women newly diagnosed with early-stage breast cancer. Cancer Nurs. 2011;34(2):E1-E13.

131. Young-McCaughan S, Mays MZ, Arzola SM, et al. Research and commentary: Change in exercise tolerance, activity and sleep patterns, and quality of life in patients with cancer participating in a structured exercise program. Oncol Nurs Forum. 2003;30(3):441-454; discussion 441-454.

132. Coleman EA, Coon S, Hall-Barrow J, Richards K, Gaylor D, Stewart B. Feasibility of exercise during treatment for multiple myeloma. Cancer Nurs. 2003;26(5):410-419.

133. Dodd MJ, Cho MH, Miaskowski C, et al. A randomized controlled trial of home-based exercise for cancer-related fatigue in women during and after chemotherapy with or without radiation therapy. Cancer Nurs. 2010;33(4):245-257.

134. Bower JE, Woolery A, Sternlieb B, Garet D. Yoga for Cancer Patients and Survivors. Cancer Control. 2005;12(3):165-171.

135. Elkins G, Fisher W, Johnson A. Mind-body therapies in integrative oncology. Curr Treat Options Oncol. 2010;11(3-4):128-140.
136. Danhauer SC, Mihalko SL, Russell GB, et al. Restorative yoga for women with breast cancer: findings from a randomized pilot study. Psychooncology. 2009;18(4):360-368.

137. Cohen L, Warneke C, Fouladi RT, Rodriguez MA, Chaoul-Reich A. Psychological adjustment and sleep quality in a randomized trial of the effects of a Tibetan yoga intervention in patients with lymphoma. Cancer. 2004;100(10):2253-2260.

138. Rosenbaum E, Gautier H, Fobair P, et al. Cancer supportive care, improving the quality of life for cancer patients. A program evaluation report. Support Care Cancer. 2004;12(5):293-301.

139. Culos-Reed SN, Carlson LE, Daroux LM, Hately-Aldous S. A pilot study of yoga for breast cancer survivors: physical and psychological benefits. Psychooncology. 2006;15(10):891-897.

140. Carson JW, Carson KM, Porter LS, Keefe FJ, Shaw H, Miller JM. Yoga for women with metastatic breast cancer: results from a pilot study. J Pain Symptom Manage. 2007;33:331-341.

141. Ortiz-Tudela E, Martinez-Nicolas A, Campos M, Rol MÁ, Madrid JA. A new integrated variable based on thermometry, actimetry and body position (TAP) to evaluate circadian system status in humans. PLoS Comput Biol. 2010;6(11):e1000996.

142. Waterhouse J, Drust B, Weinert D, et al. The circadian rhythm of core temperature: origin and some implications for exercise performance. Chronobiol Int. 2005;22(2):207-225.

143. Fallon KE, Fallon SK, Boston T. The acute phase response and exercise: court and field sports. Br J Sports Med. 2001;35(3):170-173.

144. Steensberg A, Fischer CP, Keller C, Møller K, Pedersen BK. IL-6 enhances plasma IL-1ra, IL-10, and cortisol in humans. Am J Physiol Endocrinol Metab. 2003;285(2):E433-E437.

145. Petersen AM, Pedersen BK. The role of IL-6 in mediating the anti-inflammatory effects of exercise. J Physiol Pharmacol. 2006; 57 Suppl 10:43-51.

146. Petersen AM, Pedersen BK. The anti-inflammatory effect of exercise. J Appl Physiol. 2005;98(4):1154-1162.

147. Santos RVT, Tufik S, De Mello MT. Exercise, sleep and cytokines: is there a relation? Sleep Med Rev. 2007;11(3):231-239.

148. Mistlberger RE, Skene DJ. Nonphotic entrainment in humans? J Biol Rhythms. 2005;20(4):339-352.

149. Stranahan AM, Lee K, Mattson MP. Central mechanisms of HPA axis regulation by voluntary exercise. Neuromolecular Med. 2008;10(2): 118-127.

150. Sandercock GR, Bromley PD, Brodie DA. Effects of exercise on heart rate variability: inferences from meta-analysis. Med Sci Sports Exerc. 2005;37(3):433-439.

151. Spielman AJ, Yang CM, Glovinsky PB. Assessment techniques for insomnia. In: Kryger MH, Roth T, Dement WC, editors. Principles and Practice of Sleep Medicine. Philadelphia: Elsevier; 2011:1632-1645.
Nature and Science of Sleep

\section{Publish your work in this journal}

Nature and Science of Sleep is an international, peer-reviewed, open access journal covering all aspects of sleep science and sleep medicine, including the neurophysiology and functions of sleep, the genetics of sleep, sleep and society, biological rhythms, dreaming, sleep disorders and therapy, and strategies to optimize healthy sleep. The journal welcomes

\section{Dovepress}

original research, clinical \& epidemiological studies, reviews \& evaluations, case reports and extended reports. The manuscript management system is completely online and includes a very quick and fair peerreview system, which is all easy to use. Visit http://www.dovepress.com/ testimonials.php to read real quotes from published authors. 